\title{
Thermal Design Of T/R Modules In Airborne Phased Array Antenna
}

\author{
Zhang wenxing $^{1,2}$, Wu qiang ${ }^{1,2}$, Zhao shuwei ${ }^{1,2}$ \\ 1. The 41st Research Institute of CETC \\ 2. Science and Technology on Electronic Test \& Measurement Laboratory \\ Qingdao, China \\ zwxhit@163.com
}

Keywords: Thermal simulation; T/R modules; Heat pipe; Flotherm.

\begin{abstract}
The research on the phased array radar used in airborne is very hot. Transmit/Receive(T/R) modules are the key components of the phased array radar. The performances of $T / R$ modules affect the whole performance of the phased array radar system directly. According to the characteristics of the phased array radar used in airborne, the T/R modules with low weight and high power is demanded. $\mathrm{Al} / \mathrm{Si}$ alloy is used for the cavity and the heat pipe is fixed on the back of the cavity in order to take away the heat from chips. Then the apparatus is analyzed based on Flotherm software to the thermal, Simulations show that the measures meet the requirements for stable work in thermal environments.
\end{abstract}

\section{Introduction}

With the rapid development of the technology of the phased array, the research on the phased array radar used in airborne is very hot in many countries. Transmit/Receive(T/R) modules are the foundation of the phased array. The performances of $T / R$ modules such as weight, volume and price affect the whole performance of the phased array radar system directly ${ }^{[1,2]}$. On account of the limitation that the distance of between the antenna elements is half of a wavelength, Transmit/Receive (T/R) modules which contain many components are small in size ${ }^{[3,4]}$. This results in a great deal of heat. Heat maybe damages the components if heat cannot be radiated quickly. The temperature affects components’ performance directly. Researches indicate that $70 \%$ damages of chip are caused by high-temperature; meanwhile high-temperature can shorten the life of components ${ }^{[5]}$.In order make the system work properly, the heat must be taken away from the module quickly ${ }^{[6]}$. Reasonable and effective radiator cooling system is an important part in designing. Finite Element Analysis (FEA) software can simulate process of heat from chips radiating and show the picture of temperature distribution. According to the result of thermal simulation T/R modules would be designed radiating quickly.

\section{Heat transfer process}

In $T / R$ module heat transfer process, heat is radiated by thermal conduction. When interior of a part or two contacting parts own different temperatures, relative displacement is not existed in every part of object, heat is transferred depending on the movement of molecules, atom sand free electrons, this is thermal conduction. Heat is transferred from high-temperature part to low-temperature contacting part. Basic regular pattern of thermal conduction is Eq. $1^{[7,8]}$.

$$
\mathrm{Q}=-\lambda \mathrm{A} \frac{d t}{d n}
$$

In Eq. 1, Q-thermal flux, heats go through fixed area per unit time, unit is $\mathrm{w}$; $\lambda$ - thermal conductivity, unit is $\mathrm{w} /(\mathrm{m} \bullet \mathrm{k})$; $\mathrm{dt} / \mathrm{dn}$ - temperature gradient. A- Conducting area, unit is $\mathrm{m}^{2}$. The higher thermal conductivity is, the quicker heat can be transferred. 


\section{Thermal design}

Thermal design is an important part in the designing. Firstly, find the heat source and make sure the position of heat source is good for heat dissipation. Then the material of structure meets the requirement of system .Thirdly advanced cooling measure is designed in system such as heat pipe, fan. Last but not least, thermal simulation or experiment should be performed to check the thermal design.

\subsection{Heat source}

Transmit module is the largest heat source in the system. Therefore, designing $T / R$ modules is focus on designing Transmit module. The model of Transmit module is shown in Figure 1. According to the system requirements, there are eight transceiver channels in the Transmit module. Each channel contains a MMIC chip (2 in Fig.1) whose heating power is the highest in the module. The heating-power of chip is $0.8 \mathrm{w}$. The main heat is focused on nearby place where chip is fixed. The temperature of chip is the highest point in the whole module. The keystone of the thermal design is to lay chips reasonable and take away the heat quickly.
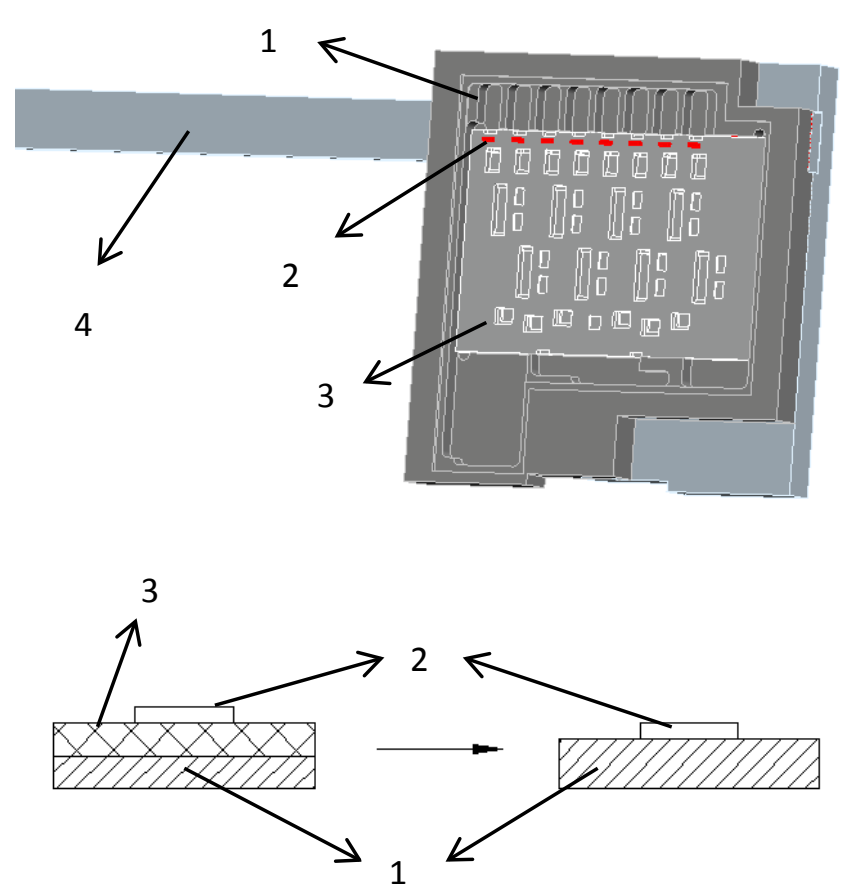

Figure 1: Model of T/R modules
According to the system requirements, Transmit module is sealed. The Majority of heat from chips is conducted to LTCC (3 in Fig.1) and then to cavity (1 in Fig.1) by solder. Surface of modules radiate by natural convection. The efficiency of natural convection is very low. It becomes especially important to create a radiating channel for avoiding the chips to be damaged.

The position of the heat source affects the cooling effect directly. It is better to lay heat source decentralized by free convection. And lay heat source near the cooling equipment contributes to taking away heat quicker. So the chips are laid on the line of the cooling equipment to improve the efficiency of heat transfer.

According to the Eq. 1, it is better that the dn is shorter. The $\mathrm{dn}$ is the distance between hot side and cold side. As shown in Fig.1, removing the chips from LTCC to cavity decreases the distance between heat source and outside. The heat transfers from chips to cavity directly.

\subsection{Material of cavity}

As we all kwon, metal will expand when temperature rises up. To avoiding chips be damaged by the cavity expansion when temperature of module rises up, the coefficient of thermal expansion (CTE) of the material of cavity is similar to chips'. The material of chips is Gallium Arsenide. And the CTE of Gallium Arsenide is $6 \times 10-6 \mathrm{~K}^{-1}$. What's more, as the $T / R$ modules are used in airborne, the weight of the $T / R$ modules is the less the better. The system requires weight of module less than 50g. It is important to ensure that the thermal conductivity (TC) of cavity is higher than LTCC. The material of cavity should have light weight-high thermal conductivity and low expansion coefficient. Conventional materials are difficult to meet the requirements above. Metal matrix composite integrates the advantages of both the matrix materials and the component materials and will achieve these goals.

\begin{tabular}{|l|l|l|}
\hline property & number & unit \\
\hline Density & 2.4 & $\mathrm{~g} / \mathrm{cm}^{3}$ \\
\hline CTE & $7.4 \times 10^{-6}\left(25^{\circ} \mathrm{C}\right)$ & $\mathrm{K}^{-1}$ \\
\hline TC & 120 & $\mathrm{~W} / \mathrm{m} \cdot \mathrm{K}$ \\
\hline
\end{tabular}

Table 1: The physical properties of Al-Si alloy ${ }^{[9]}$

Hyper-eutectic Al-Si alloy has a series of advantages, such as small specific gravity, good thermal conductivity ,good 
corrosion resistance, low expansion coefficient and excellent processing properties. The physical properties of Al-Si alloy shown in Table 1 meet the requirements of T/R modules.

\subsection{Heat pipe}

At present, the thermal design technology has developed maturely and practically. The thermal design technology mainly includes:

1) Free convection cooling

2) Forced convection cooling

3) Evaporation cooling

4) Heat pipe technologies

5) Others, such as Cold plate and Micro-channel cooling system.

Every cooling method has advantage and disadvantage. Free convection cooling suits to the equipment used in large heat dissipation space with low power. Forced convection cooling, evaporation cooling and heat pipe suit to the equipment used in limited space with high power. The heat flux density of the equipment and the safety temperature of the electronic decide the heat dissipation method.

In model, two Transmit modules are fixed back to back as showed in Fig 1.The area of dissipation is $72 \mathrm{~cm}^{2}$.the heat flux density is $0.18 \mathrm{~W} / \mathrm{cm}^{2}$. By analyzing several cooling method and comparing their advantages and shortcoming, the heat pipe is suitable for this Transmit module.

Heat Pipe is advanced heat dissipation equipment, which consists of shell, pipe core and media. The thermal conductivity of the heat pipe is higher than any metal or alloy has been known. Heat conduction and phase transformation make the heat pipe take away the heat from the heat source to outside quicker. The heat pipe (4 in Fig.1) is fixed in the slot that back in cavity under chips. The slot not only reduces the weight but also the thermal resistance of the module.

\section{Thermal simulation based on Flotherm}

\subsection{Model and simulation}

Model of Transmit module externalized of SAT can be imported into Flotherm, then the model need to be healed 、 voxelized and transferred MCAD ${ }^{[10]}$. All bodies would be attached material (by Table 2). The conductivity of heat pipe is $1000 \mathrm{w} /(\mathrm{m} \bullet \mathrm{k})$.In the model, the conductivity of copper is set 2000 to represent heat pipe. Ambient temperature is $50^{\circ} \mathrm{C}$, and cold part of heat pipe is $50^{\circ} \mathrm{C}$, chips also are set thermal. Size of solder is $2 * 3 * 0.3$, so minimum mesh size of model should be less than 0.3 . Total number of cell is 848232.

\begin{tabular}{|l|l|l|}
\hline Name & Material & $\begin{array}{l}\text { Conductivity } \\
{[\mathrm{w} /(\mathrm{m} \bullet \mathrm{k})]}\end{array}$ \\
\hline Cavity & Silumin & 164 \\
\hline LTCC & Aluminum & 160 \\
\hline Chip & Gallium Arsenide & 48 \\
\hline Heat pipe & Copper & 2000 \\
\hline
\end{tabular}

Table 2: Material introduction

Gravity direction is set according actual placed direction. Considering effect of heat pipe, both model with heat pipe and model without heat pipe are simulated as contrary. In the model without heat pipe, the conductivity of copper is set 380. Two monitor points are attached to chips to monitor temperature changing.

\subsection{Result and analysis}

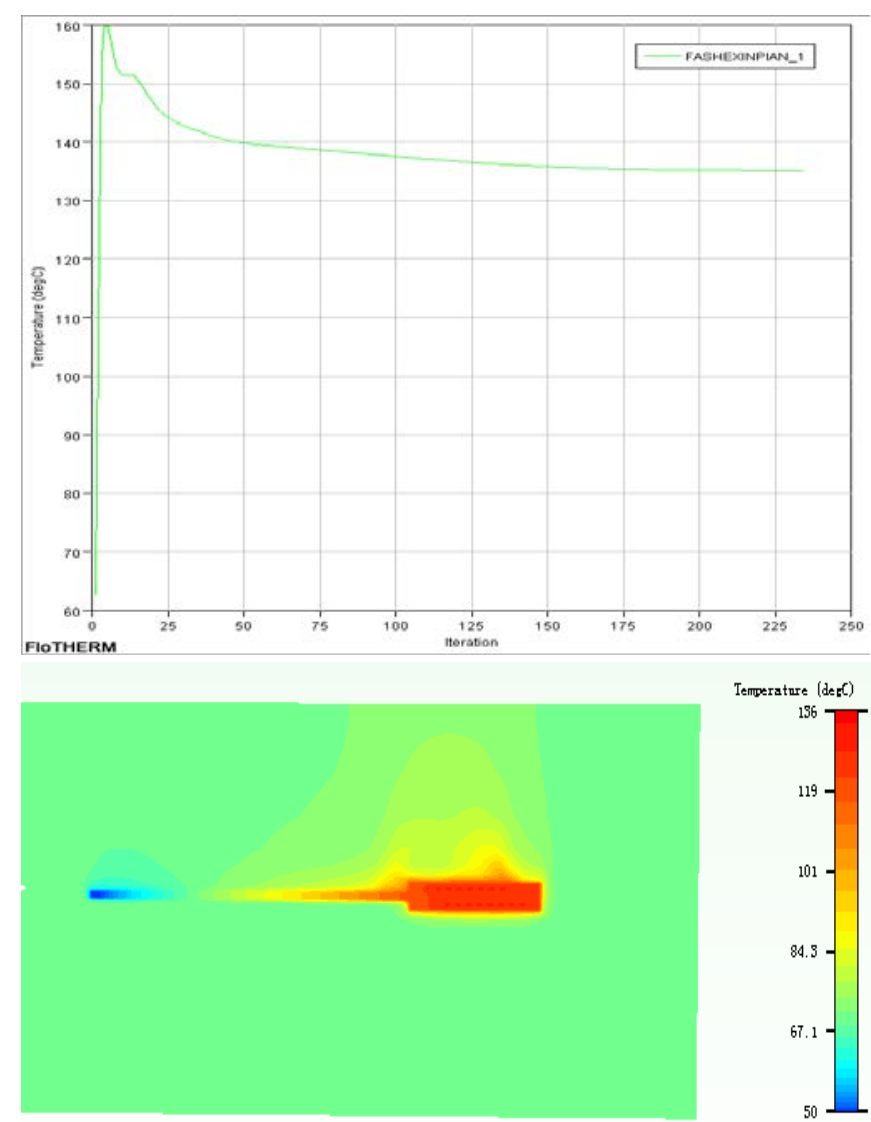

Figure 2: Simulation result of model without heat pipe 


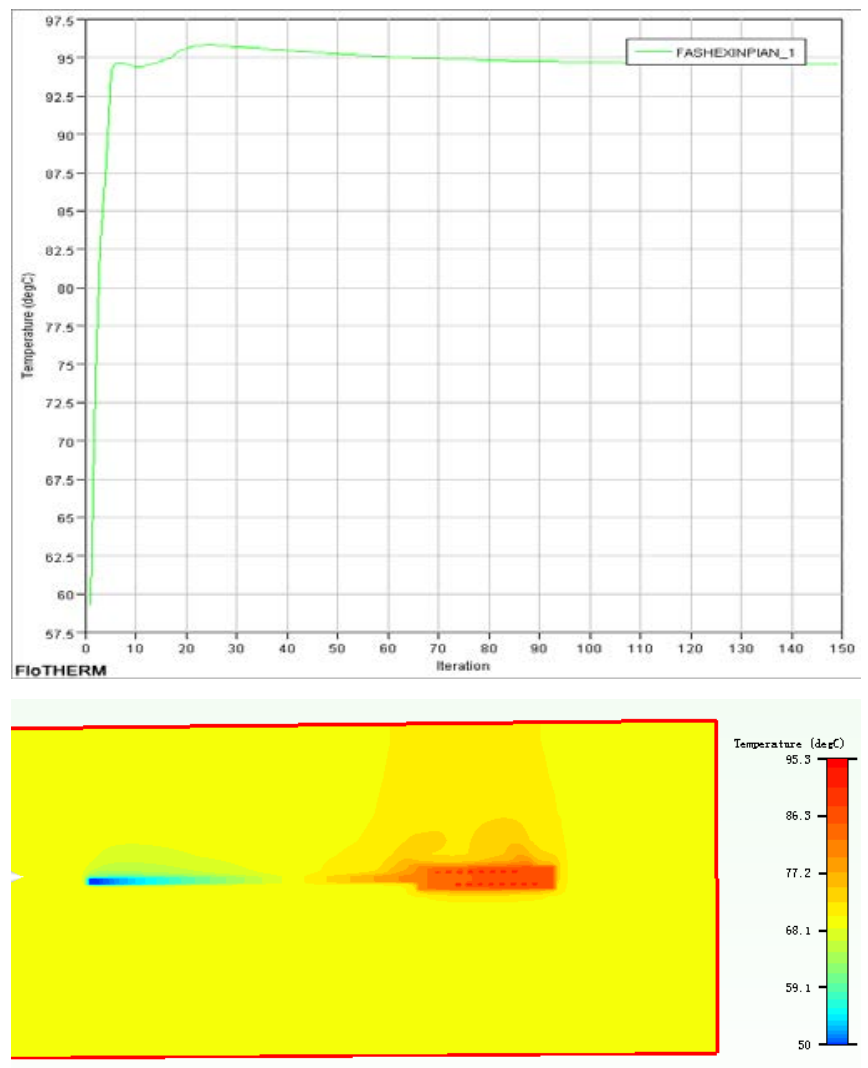

Figure 3: Simulation result of model with heat pipe

The simulation result is shown above. The highest temperature of whole unit without heat pipe is $136^{\circ} \mathrm{C}$ shown in Figure 2. And the highest temperature of whole unit with heat pipe is $95^{\circ} \mathrm{C}$ shown in Figure 3. Heat pipe cooling system can cool the whole unit effectively. The maximum normal using temperature of the chip is $130{ }^{\circ} \mathrm{C}^{[7]}$, and industry ambient temperature usually higher than safe temperature $30^{\circ} \mathrm{C}$, so $95^{\circ} \mathrm{C}$ is security to chip work normally. Therefore cooling system with heat pipe contributes to guaranteeing chip work steadily and prolonging chip's lifetime.

\section{Conclusions}

This paper proposes a thermal design of T/R modules, which is an important part in the designing. According to phased array radar used in airborne, T/R modules are small-light and high-power. Al-Si alloy is the material of cavity that makes modules light and low expansion coefficient. Radiator system using heat pipe is very effective, that has been proved by the thermal simulation. Radiator system in this paper provides reference for engineering designers and researchers.

\section{Acknowledgements}

The author wishes to thank funding organizations for providing this template and Ma jianzhuang for providing technical support and assistances.

\section{References}

[1] E. Brookner. Phased arrays for the new millennium.2000 IEEE International Conference on Phased Array Systems and Technology, 2002, 3-13.

[2] E li Brookner. Phased Arrays And Radars - Past. Present and Future. Microwave J. 2006,149(1):24-41.

[3] WEI Tao: Thermal Simulation of an Airborne Millimeter-wave Radar Antenna. Electro - Mechanical Engineering. Jun. 2014.

[4] TANG Xiaoyun. Summarization of Phased Array Radar Seeker [J]. Aero Weaponry, 2013(3) : 25-30.

[5] Xie Deren: Thermal design of electric Unit: Southeast University press. Nanjing.2001

[6] Lu Shen gang: Thermal Design for $T / R$ Module of Active Phased Array Radar. 2009 Mechanical and Electronics Conference, 2009.

[7] http://global-sei.com/Electrooptic/about/office.html.

[8] Xu Weixin: Reliability design of electric product: Electric industry press. Beijing. 2000.

[9] XIE Yi Shui: Integrated Manufacturing Technology for RF Front-end of Millimeter-wave Active Phased Array. Development \&Innovation of Machinery \&Electrical Products.Nov,2013.

[10] Mentor company .thermal optimal design of electric unit base on Flotherm. CAD/CAM and manufacturing industry information.2010.11. 